University of Texas at El Paso

ScholarWorks@UTEP

$1-2014$

\title{
From Global to Local Constraints: A Constructive Version of Bloch's Principle
}

Martine Ceberio

The University of Texas at El Paso, mceberio@utep.edu

Olga Kosheleva

The University of Texas at El Paso, olgak@utep.edu

Vladik Kreinovich

The University of Texas at El Paso, vladik@utep.edu

Follow this and additional works at: https://scholarworks.utep.edu/cs_techrep

Part of the Computer Sciences Commons

Comments:

Technical Report: UTEP-CS-14-08

Published in Proceedings of the Seventh International Workshop on Constraints Programming and Decision Making CoProd'2014, Wuerzburg, Germany, September 21, 2014.

\section{Recommended Citation}

Ceberio, Martine; Kosheleva, Olga; and Kreinovich, Vladik, "From Global to Local Constraints: A Constructive Version of Bloch's Principle" (2014). Departmental Technical Reports (CS). 816.

https://scholarworks.utep.edu/cs_techrep/816

This Article is brought to you for free and open access by the Computer Science at ScholarWorks@UTEP. It has been accepted for inclusion in Departmental Technical Reports (CS) by an authorized administrator of ScholarWorks@UTEP. For more information, please contact Iweber@utep.edu. 


\title{
From Global to Local Constraints: A Constructive Version of Bloch's Principle
}

\author{
Martine Ceberio, Olga Kosheleva, and Vladik Kreinovich \\ University of Texas at El Paso, El Paso, TX 79968, USA \\ \{mceberio, olgak, vladik\}@utep.edu
}

\begin{abstract}
Generalizing several results from complex analysis, A. Bloch formulated an informal principle - that for every global implication there is a stronger local implication. This principle has been formalized for complex analysis, but is has been successfully used in other areas as well. In this paper, we propose a new formalization of Bloch's Principle, and we show that in general, the corresponding localized version can be obtained algorithmically.
\end{abstract}

\section{Bloch's Principle: Formulation of the Problem}

Bloch's Principle: a brief history (see [4] for details). Liouville's Theorem states that every analytical function $f(z)$ which is bounded on a whole complex plane and for which $f(0)=0$ is equal to 0 ; see, e.g., [3]. This theorem requires that the constraint $|f(z)| \leq C$ be satisfied globally, i.e., for all $z$. What if this constraint is only satisfied locally, i.e., for all $z$ from a bounded set? Such a "localization" of Liouville's theorem was indeed proven by H. A. Schwarz: if a function $f(z)$ for which $f(0)=0$ is analytical for all $z$ from a disk

$$
B_{R}(0) \stackrel{\text { def }}{=}\{z:|z|<R\}
$$

and $|f(z)| \leq C$ for all $z \in B_{R}(0)$, then for all such values $z$, we get $|f(z)| \leq \frac{C}{R} \cdot|z|$. When the size $R$ increases, the bound tends to 0 ; so for $R \rightarrow \infty$, we get Liouville's Theorem.

Several similar localizations of global results are known. In 1926, A. Bloch, formulated a general (informal) Bloch's Principle: that for every global result, there is a local version from which this global result follows [2]. In complex analysis, this principle was formalized; however, there are many interesting results of the use of Bloch's Principle in other areas of mathematics.

Problem. Can we formalize Bloch's Principle in a context which is more general than complex analysis? If yes, and if the appropriate the localization always exists, can we find it algorithmically?

What we do in this paper. In this paper, we provide positive answers to both questions.

Comment. Of course, due to the informal character of Bloch's principle, no answer is final - it is always possible that our result (or a similar result) holds in a more general context. 


\section{Bloch's Principle: General Formalization}

Analysis of the problem. In general terms, the Liouville's theorem has the form

$$
\forall f \in \mathcal{F}(\forall x(f(x) \in A(x)) \Rightarrow \forall x(f(x) \in B(x))),
$$

where $\mathcal{F}$ is the class of all analytical functions for which $f(0)=0, x$ goes over all complex numbers, $A(x)=\{x:|x| \leq C\}$ is the set of all the valued bounded by the given constant $C$, and $B(x)=\{0\}$.

The implication (1) says that if the constraint $f(x) \in A(x)$ is exactly satisfied for all possible values $x$, then the conclusion holds. What we want to prove is that when the constraint is "approximately" satisfied - i.e., if it satisfied with some accuracy $\delta>0$ for all the values $x$ which are at a distance $r$ form 0 - then the conclusion is also approximately satisfied, with some accuracy $\varepsilon>0$ and for all values at a distance $R$ from 0 . We also want to make sure that when $\delta \rightarrow 0$ and $r \rightarrow \infty$, then $\varepsilon \rightarrow 0$ and $R \rightarrow \infty$. In other words, we want to prove that for every $\varepsilon>0$ and $R>0$, there exist $\delta>0$ and $r>0$ for which, if the condition is satisfied with accuracy $\delta$ for all $x$ which are $r$-close to 0 , then the conclusion is satisfied with accuracy $\varepsilon$ for all $x$ which are $R$-close to 0 .

A natural way to describe the fact that $f(x)$ is "approximately" in the set $A(x)$ (or in the set $B(x)$ ) is to say that $f(x)$ is close to the set $A(x)$ in the sense of the usual distance $d(z, S) \stackrel{\text { def }}{=} \inf \{d(z, s): s \in S\}$. In the above case, the sets $A(x)$ and $B(x)$ are compact, so $d(z, A(x))=0$ if and only if $z \in A(x)$. Thus, the global result (1) can be reformulated in the equivalent form

$$
\forall f(\forall x d(f(x), A(x))=0 \Rightarrow \forall x d(f(x), B(x))=0)) .
$$

and the desired localized result has the form

$$
\begin{gathered}
\forall \varepsilon>0 \forall R>0 \exists \delta>0 \exists r>0 \\
\forall f\left(\left(\forall x\left(d\left(x, x_{0}\right) \leq r \Rightarrow d(f(x), A(x)) \leq \delta\right)\right) \Rightarrow\right. \\
\left.\left(\forall x\left(d\left(x, x_{0}\right) \leq R \Rightarrow d(f(x), B(x)) \leq \varepsilon\right)\right)\right) .
\end{gathered}
$$

It is worth mentioning that in the case of Liouville's Theorem (and in several similar results mentioned in [4]), not only all the sets $A(x)$ and $B(x)$ compact, but they also continuously depend on $x$ - in the sense of the Hausdorff metric $d_{H}(A, B) \stackrel{\text { def }}{=} \max \left(\max _{a \in A} d(a, B), \max _{b \in B} d(b, A)\right)$.

The class $\mathcal{F}$ is also compact in some reasonable sense: indeed, for ever bounded set $D$, the set of all these functions limited to $D$ is compact in the usual metric $d_{D}(f, g)=\max _{x \in D} d(f(x), g(x))$. Indeed, for an analytical function $f(z)$, its value $f(z)$ can be described by a Cauchy integral over a surrounding curve $\gamma: f(z)=\int_{\gamma} \frac{f(t)}{z-t} d t$. Differentiation of this formula enables us to get a similar formula for the derivative $f^{\prime}(z)$. Thus, when the analytical function is 
bounded, its derivative is also bounded. Due to Ascoli-Arzela theorem, this implies that the corresponding class of functions is compact - when limited to each bounded domain.

It is also important to notice that the notion of an analytical function is locally defined, in the sense that if a function $f(x)$ coincides with some analytical function in every neighborhood, then it is analytical itself.

Thus, we arrive at the following natural formalization of Bloch's Principle.

Definition 1. Let us call a metric space bounded-compact if every closed bounded set in this space is compact.

Comment. In particular, this implies that every closed ball

$$
B_{r}\left(x_{0}\right) \stackrel{\text { def }}{=}\left\{x: d\left(x, x_{0}\right) \leq r\right\}
$$

is compact. Vice versa, if for some point $x_{0}$, every closed ball with a center at $x_{0}$ is compact then every closed bounded set is compact too: indeed, very bounded set is contained in some ball $B_{r}\left(x_{0}\right)$, and a closed subset of a compact set is also compact.

Definition 2. Let $\mathcal{F}$ be a class of functions from a bounded-compact metric space $X$ to a bounded-compact metric space $Y$. We say that the class $\mathcal{F}$ is boundedcompact if for every compact set $K \subset X$, this class is compact in the metric $d_{K}(f, g) \stackrel{\text { def }}{=} \sup _{x \in K} d(f(x), g(x))$.

Definition 3. Let $\mathcal{F}$ be a class of a functions from $X$ to $Y$, and let $x_{0}$ be a point in $x_{0}$. We say that a function $f: X \rightarrow Y$ locally belongs to the class $\mathcal{F}$ if for every $n$, there exists a function $f_{n} \in \mathcal{F}$ which coincides with $f$ on $B_{n}\left(x_{0}\right)$.

Comment. This definition uses the point $x_{0}$, but one can easily check that the resulting notion does not depend on $x_{0}$.

Definition 4. We say that a bounded-compact class of functions $\mathcal{F}$ is locally defined if it contains all the functions that locally belong to this class.

Definition 5. Let $\mathcal{F}$ be a bounded-compact locally defined class of functions. By an $\mathcal{F}$-constraint $A$, we mean a (Hausdorff)-continuous function that map each point $x \in X$ into a compact set $A(x) \subseteq Y$.

Definition 6. Let $\mathcal{F}$ be a bounded-compact locally defined class of functions, and let $A$ and $B$ be $\mathcal{F}$-constraints.

- We say that the constraint $A$ globally implies the constraint $B$ if for every function $f \in \mathcal{F}$, the condition $\forall x(f(x) \in A(x))$ implies $\forall x(f(x) \in B(x))$.

- We say that the constraint $A$ locally implies the constraint $B$ for $\varepsilon, R, \delta$, and $r$ if for every function $f(x)$ for which $d(f(x), A(x)) \leq \delta$ for all $x$ with $d\left(x, x_{0}\right) \leq r$, we have $d(f(x), B(x)) \leq \varepsilon$ for all $x$ with $d\left(x, x_{0}\right) \leq R$.

- We say that the constraint $A$ locally implies the constraint $B$ if for every $\varepsilon>0$ and for every $R>0$, there exist real numbers $\delta>0$ and $r>0$ such that $A$ locally implies $B$ for $\varepsilon, R, \delta$, and $r$. 
Comment. The definition of local implication uses a point $x_{0}$, but one can easily see that the corresponding property does not change if we replace this point with any other point from the metric spaces $X$.

Proposition 1. Let $\mathcal{F}$ be a bounded-compact locally defined class of functions, and let $A$ and $B$ are $\mathcal{F}$-constraints. Then, if $A$ globally implies $B$, then $A$ locally implies $B$.

Proof. We will prove the result by contradiction. Let us assume that $A$ does not locally imply $B$. This means that there exist $\varepsilon>0$ and $R>0$ such that for every $n$, there is a function $f_{n} \in \mathcal{F}$ for which $\max _{x \in B_{n}\left(x_{0}\right)} d\left(f_{n}(x), A(x)\right) \leq 1 / n$ but $d\left(f_{n}\left(x_{n}\right), B\left(x_{n}\right)\right)>\varepsilon$ for some $x_{n} \in B_{x_{0}}(R)$. Since the sequence $x_{n}$ is contained in a compact set $B_{R}\left(x_{0}\right)$, it has a subsequence which converges to some limit $\ell$. Without losing generality, we can assume that $x_{n} \rightarrow \ell$.

Since $\mathcal{F}$ is compact relative to each metric $d_{B_{k}\left(x_{0}\right)}$, from the sequence $f_{n}$, we can extract a subsequence $n(1, i)$ convergent for $k=1$; from this subsequence, we can extract a subsequence $n(2, i)$ which is convergent for $k=2$, etc. The diagonal subsequence $f_{n(i, i)}$ then converges for all $k$. This convergence is for all $x$, no matter how far from $x_{0}$ we are, so we can defining a point-wise limit function $f(x)$. On each ball $B_{k}\left(x_{0}\right)$, this limit coincides with the corresponding limit from $\mathcal{F}$ limited to this ball. Thus, the limit function $f(x)$ locally belongs to $\mathcal{F}$; since the class $\mathcal{F}$ is locally defined, this means that $f \in \mathcal{F}$.

For the limit function $f$, for every $x$, the condition $d\left(f_{n}(x), A(x)\right) \leq 1 / n$ in the limit tends to $d(f(x), A(x))=0$. Since $A$ globally implies $B$, we conclude that we have $d(f(x), B(x))=0$ for all $x$, in particular, that we have $d(f(\ell), B(\ell))=0$. However, from $d\left(f_{n}\left(x_{n}\right), B\left(x_{n}\right)\right)>\varepsilon$, in the limit $x_{n} \rightarrow \ell$, we get $d(f(\ell), B(\ell)) \geq$ $\varepsilon>0$. This contradictions shows that our assumption is wrong, and $A$ does locally imply $B$. The proposition is proven.

\section{Bloch's Principle: A Constructive Version}

Towards an algorithmic version. In this paper, we will use the usual definitions of computable numbers, functions, compact spaces, etc.; see, e.g., $[1,5]$.

Proposition 2. If spaces $X$ and $Y$ are computable and computably boundedcompact, and if $A$ and $B$ are computable functions for which $A$ globally implies $B$, then there exists an algorithm that, given rational numbers $\varepsilon>0$ and $R>0$, produces computable numbers $\delta>0$ and $r>0$ for which $A$ locally implies $B$ for $\varepsilon, R, \delta$, and $r$.

Proof. From the proof of Proposition 1, we can conclude that that for $\varepsilon_{0}=\varepsilon / 3$ and for $R_{0}=R+1$, there exists an integer $n=n_{0}$ for which $r=n$ and $\delta=1 / n$ satisfy the desired property. Let us show how to algorithmically find this $n$. For that, we will repeat the below computations for $n=1,2, \ldots$ until we find the value $n$ for which the desired condition is satisfied.

In these computations, we will use the fact that there are algorithms for computing the maximum and minimum of a computable function over a computable 
compact. We will also use the fact that for a computable function $F(x)$ on a computable compact set $K$, for every two computable numbers $z^{-}<z^{+}$within the range of $F(x)$ on $K$, we can compute an intermediate value $z \in\left(z^{-}, z^{+}\right)$for which the set $\{x: F(x) \leq z\}$ is a computable compact.

Before we go through $n=1,2, \ldots$, we use the intermediate-value algorithm to compute a value $R^{\prime} \in(R, R+1)$ for which the ball $B_{R^{\prime}}\left(x_{0}\right)$ is computably compact.

Then, for each $n$, we compute a value $r_{n} \in(n-1, n)$ for which the closed ball $B_{r_{n}}\left(x_{0}\right)$ is a computable compact. Since this ball is a computable compact, the value $v(f) \stackrel{\text { def }}{=} \max _{x \in B_{r_{n}}\left(x_{0}\right)} d(f(x), A(x))$ is also computable - and is, therefore, a computable function of $f \in \mathcal{F}^{\prime} \stackrel{\text { def }}{=} \mathcal{F}_{\mid B_{x_{0}}(R)}$.

The restriction $\mathcal{F}^{\prime}$ is a computable compact. Thus, by the same intermediatevalue result, we can compute a value $\delta_{n} \in(1 / n, 1 /(n-1))$ for which the set $S \stackrel{\text { def }}{=}\left\{f: v(f) \leq \delta_{n}\right\}$ is a computable compact. We can therefore compute the maximum $M$ of a computable function $d(f(x), B(x))$ over all $x \in B_{R^{\prime}}\left(x_{0}\right)$ and all $f \in S$ with any given accuracy. Let us compute it with accuracy $\varepsilon / 3$. If the resulting estimate $\widetilde{M}$ is $\leq(2 / 3) \cdot \varepsilon$, we stop.

Let us show that if we stop, then we get the desired $n$. Indeed, in this case, if for some $f$, we have $d(f(x), A(x)) \leq 1 / n<\delta_{n}$ for all $x \in B_{n}\left(x_{0}\right)$, then (since $\left.r_{n}<n\right)$ this inequality is also true for all $x \in B_{r_{n}}\left(x_{0}\right)$, hence $v(f)<\delta_{n}$. Every $x \in B_{R}\left(x_{0}\right)$ belongs to $B_{R^{\prime}}\left(x_{0}\right)$ and thus, for this $x$, we have $d(f(x), B(x)) \leq M$. Since $M \leq \widetilde{M}+\varepsilon / 3$ and $\widetilde{M} \leq(2 / 3) \cdot \varepsilon$, we conclude that $d(f(x), B(x)) \leq \varepsilon$.

Let us now show that the above algorithm will stop for $n=n_{0}+1$. By definition of $n_{0}$, if $x \in B_{n_{0}}\left(x_{0}\right)$ and $d(f(x), A(x)) \leq 1 / n_{0}$, then $d(f(x), B(x)) \leq \varepsilon / 3$ for all $x \in B_{R_{0}}\left(x_{0}\right)$. Here, $R^{\prime}<R+1=R_{0}$, so $x \in B_{R^{\prime}}\left(x_{0}\right)$ implies that $x \in B_{R_{0}}\left(x_{0}\right)$. Similarly, since $r_{n}>n-1=n_{0}$, we conclude that $\max _{x \in B_{n_{0}}\left(x_{0}\right)} d(f(x), A(x)) \leq v(f)=\max _{x \in B_{r_{n}}\left(x_{0}\right)} d(f(x), A(x))$ and thus, $v(f) \leq \delta_{n}<$ $1 / n_{0}$ implies that $\max _{x \in B_{n_{0}}\left(x_{0}\right)} d(f(x), A(x))<\frac{1}{n_{0}}$. Thus, indeed, for all such $x$ and $f$, we have $d(f(x), B(x)) \leq \varepsilon / 3$; hence, the largest value $M$ is $\leq \varepsilon / 3$, so $\widetilde{M} \leq(2 / 3) \cdot \varepsilon$, and the algorithm will stop. The proposition is proven.

Acknowledgments. This work was supported in part by the National Science Foundation grants HRD-0734825, HRD-124212, and DUE-0926721.

\section{References}

1. E. Bishop and D. S. Bridges, Constructive Analysis, Springer, New York, 1985.

2. A. Bloch, "La conception actuelle de la theorie de fonctions entieres et meromorphes", Enseignement mathematique, 1926, Vol. 25, pp. 83-103.

3. S. Lang, Complex Analysis, Springer Verlag, New York, 2003.

4. R. Osserman, "From Schwarz to Pick to Ahlfors and beyond", Notices of the American Mathematical Society, 1999, Vol. 46, No. 8, pp. 868-873.

5. K. Weihrauch, Computable Analysis, Springer-Verlag, Berlin, 2000. 